\title{
INTERACTIVE IRRIGATION SYSTEM THROUGH MOBILE WITH IVR RESPONSE
}

\author{
EswaraRao Pandiripalli ${ }^{1}$, Phani Ram Veeramachaneni ${ }^{2}$, SambhaniMadhu Babu ${ }^{3}$, Jafar Sadik \\ ${ }^{1,2,3}$ Usha Rama College of engineering \& technology, Telaprolu, Vijayawada, Krishna District, A.P \\ I eswar.yuvar@gmail.com, ${ }^{2}$ mailrvee@gmail.com, ${ }^{3}$ sambhanimadhubabu@gmail.com \\ ${ }^{4}$ P.V.P Siddhartha Institute of Technology, Kanuru, Vijayawada, jafarsadikle4@gmail.com
}

\begin{abstract}
Now a day's Constant electricity fluctuation and irregular power availability for irrigation are daily problems faced by many farmers for a long time. Though many farmers use diesel operated pumps, a suitable alternative, which requires neither diesel nor electricity and yet meets their irrigation requirements may be welcome. And also in home power will be wasted in so many situations due to those situations electricity bill increases. To rectify such type of problems we developed a device which is for use of controlling Motor pumps from any location. It is a mobile based remote control system ${ }^{[7]}$ for switching on/off and monitoring the water pumps, by using which a farmer or a person can be free from so many routine problems associated with timely irrigation, saving a lot of water, electricity, fuel, time, cost effective. The way of it works is, Motor Pump is connected to the device. Mobile is kept near device which can trigger the motor pump to start and stop. Whenever power comes at field then an automatic call ${ }^{[9]}$ from system to the user to indicate power status. The person has to just call to Mobile kept near to the Motor pump and has to press his code to Start or Stop the Motor with IVR ${ }^{[10]}$ response. He can also know whether the power is present or not and know the motor status.
\end{abstract}

Keywords: DTMF decoder, IVR module, Phase selector, Rain detector, Water sensor.

\section{INTRODUCTION}

Witnessing various work hazards involved in domestic cultivation and vouching the same in various areas the need for change seemed evident. Customizable IVRS module ability to accommodate usage for a set time depending on the availability of electricity. Eliminate uncertainty in power, electrical hazards.

\section{1 Overview}

- Problem

- Solution

\subsubsection{Problems of Already Existed Ones}

Problems caused due to the existed systems are doesn't provide any power status, motor on/off status. Some of the systems are purely SMS based systems. But many of farmers didn't know about SMS.

\subsubsection{Solution:}

So premier intension behind is minimizing work hazards, improving the use of irrigation, maintaining optimal usage of electricity to provide an interactive system to formers. The main aim of this project is to provide flexibility to farmers to trigger the motor pumps from any location in an easy way.

What we are aiming at to provide the options to former through our system
- Automatic intimation through calling from System to the User.

- Water pump can be controlled from any location by a mobile phone or land phone.

- One can check availability of the power supply at the pump set.

- One can check the load on/off status by an IVR response simply.

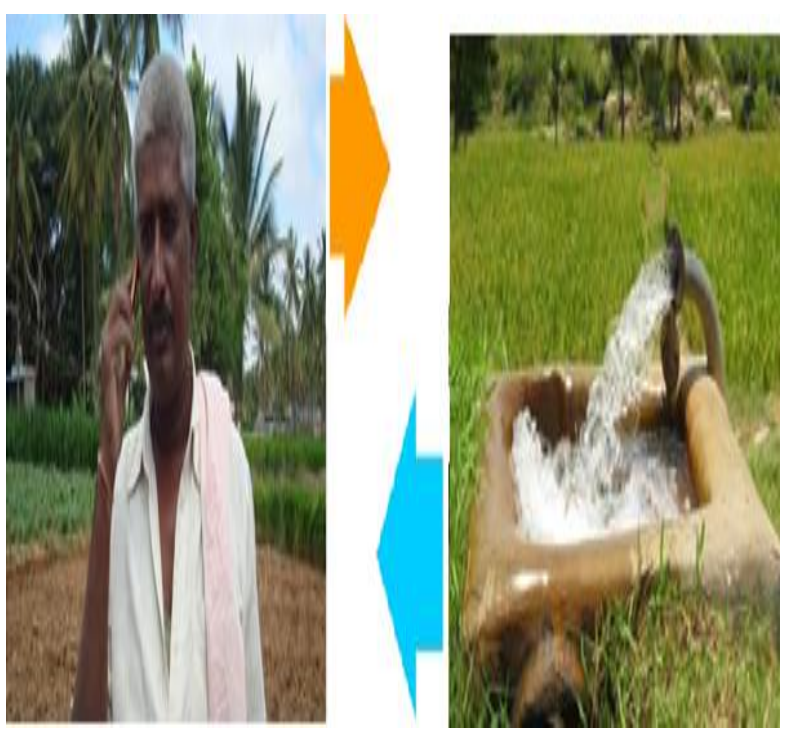

Fig1: Use of System in real-time 


\section{SYSTEM ANYLASIS}

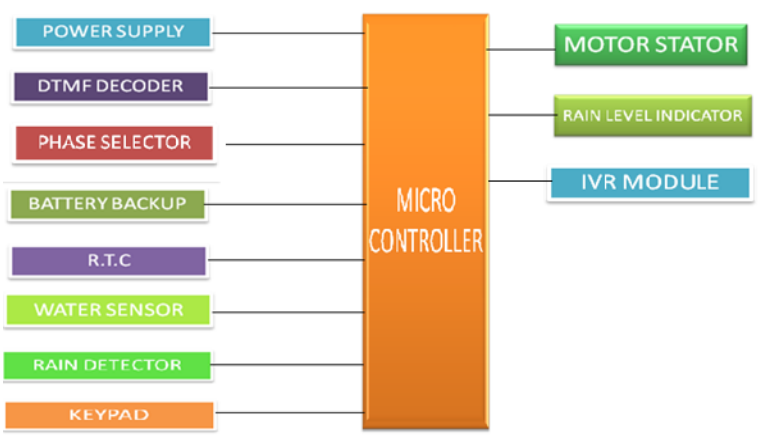

Fig2: System Block Diagram

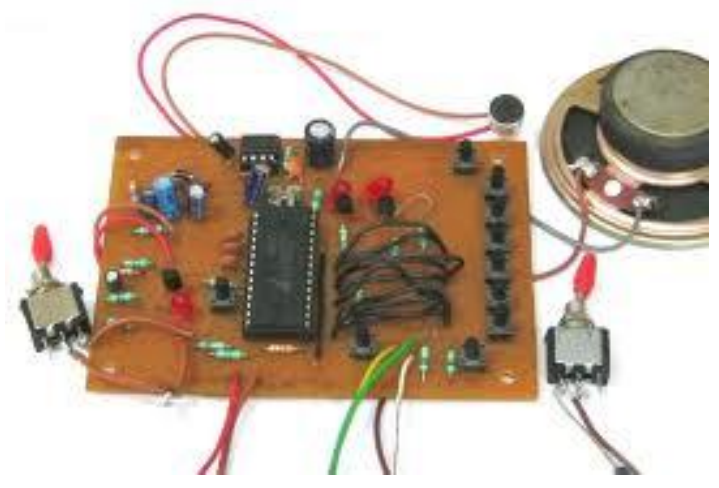

Fig3: IVR module

Whenever we make a call to the destination mobile it will be automatically lift the call. When the farmer presses the appropriate buttons in the mobile to trigger the motor pump, a DTMF tone will be released. The released DTMF tone will be decoded through DTMF decoder (MT8870). The decode data from the decoder will be sends to micro-controller. The microcontroller processes the given input data from decoder. The micro-controller propagates the appropriate signals to the relay driving circuit. Finally the relay driving circuit actuates the respective relays to control the motor pump. After perform the operation, the system gives the status report to the farmer with IVR response. The farmer also knows the rain information at field. And also set the alarm to trigger the motor pump in a fixed period of time.

\subsection{Hardware requirements:}

Components:

$\begin{array}{ll}\text { Micro Controller } & \text { : AT89S52 } \\ \text { DTMF Decoder IC } & \text { : MT8870 } \\ \text { Regulators } & : \text { LM7805, LM7810 } \\ \text { Voice recording IC } & : \text { APR6008 } \\ \text { Relays } & : \text { ULN2803 IC }\end{array}$

\subsection{Software Requirements}

KEIL $\mu$ Vision 4

NI Ulti Board

\section{A. Microcontroller (AT89S52)}

\section{Description}

The AT89S52 is a lower-power, high performance CMOS 8-bit microcomputer with 4Kbytes of flash Erasable and Programmable Read Only Memory (EPROM). The device is manufactured using Atmel's high-density non-volatile memory technology and is compatible with the industry standard MCS51 instruction set and pin out. The on-chip flash allows the program memory to be reprogrammed in-system or by a conventional non-volatile memory programmer. By combining a versatile 8-bit CPU with flash on a monolithic chip, the Atmel AT89S52 is a powerful microcontroller, which provides a highly flexible and cost effective solution to many embedded control applications.

\section{B. DTMF Decoder IC(MT8870)}

\section{Description}

The M-8870 is a full DTMF Receiver that integrates both band split filter and decoder functions into a single18-pin DIP or SOIC package. Manufactured using CMOS process technology, the M-8870 offers low power consumption $(35 \mathrm{~mW} \max )$ and precise data handling. Its filter section uses switched capacitor technology for both the high and low group filters and for dial tone $r$ ejection. Its decoder uses digital counting techniques to detect and decode all 16 DTMF tone Pairs into a 4-bit code. External component count is minimized by provision of an On-chip differential input amplifier, clock generator, and latched tri -state interface bus. Minimal external components required include a low- cost $3.579545 \mathrm{MHz}$ color burst crystal, a timing resistor, and a timing capacitor. The M-8870-02 Provides a power -down option which, when enabled, drops consumption to less than $0.5 \mathrm{Mw}$

\section{Pin Configuration:}

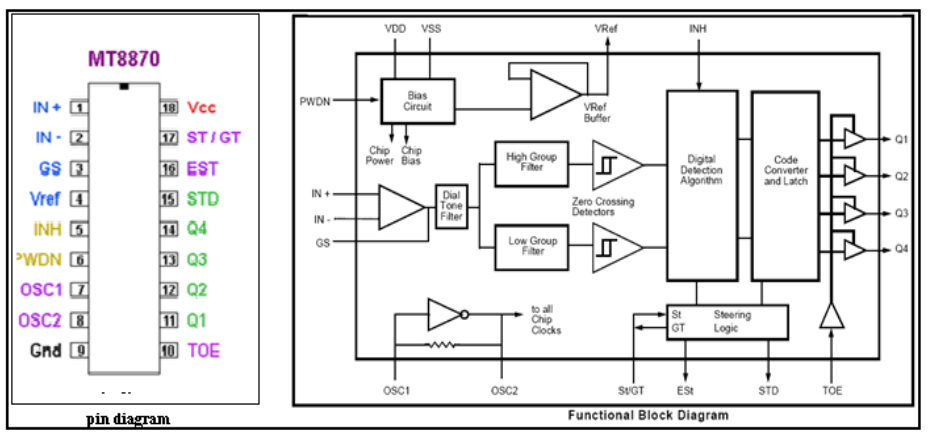

Fig4: System Block and pin Diagram 


\section{Pin Description:}

1) IN+: Non-Inverting Op-Amp (Input).

2) IN-: Inverting Op-Amp (Input).

3) GS: Gain Select. Gives access to output of front end differential amplifier for connection of feedback resistor.

4) VRef: Reference Voltage (Output). Nominally VDD/2 is used to bias inputs at mid-rail.

5) INH Inhibit (Input): Logic high inhibits the detection of tones representing characters A, B, C and D. This pin input is internally pulled down.

6) PWDN: Power down (Input). Active high. Powers down the device and inhibits the oscillator. This pin input is internally pulled down.

7) OSC1: Clock (Input).

8) OSC2: Clock (Output). A $3.579545 \mathrm{MHz}$ crystal connected between pins OSC1 and OSC2 completes the internal oscillator circuit.

9) VSS: Ground (Input). 0V typical.

10) TOE: Three State Output Enable (Input). Logic high enables the outputs Q1-Q4. This pin is pulled up internally.

11-14) Q1-Q4: Three State Data (Output). When enabled by TOE, provide the code corresponding to the last valid tone-pair received (see Table 1). When TOE is logic low, the data outputs are high impedance.

15) StD: Delayed Steering (Output).Presents logic high when a received tone-pair has been registered and the output latch updated; returns to logic low when the voltage on St/GT falls below VTSt.

16) ESt: Early Steering (Output). Pres enters logic high once the digital algorithm has detected a valid tone pair (signal condition). Any momentary loss of signal condition will cause ESt to return to a logic low.

17) St/GT: Steering Input/Guard time (Output) Bidirectional. A voltage greater than VTSt detected at Causes the device to register the detected tone pair and update the output latch. A voltage less than VTSt free the device to accept a new tone pair. The GT output acts to reset the external steering time constant; its state is a function of ESt and the voltage on St.

18 VDD Positive power supply (Input). +5V typical
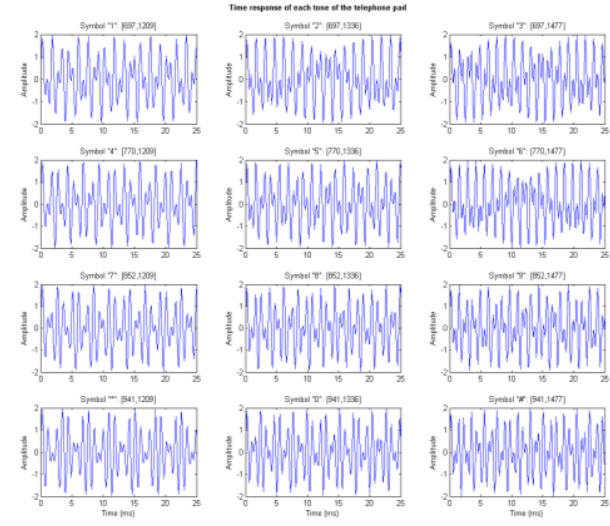

Fig 5: DTMF signals

\section{$\mu$ Vision Keil}

KEIL $\mu$ Vision is the name of software dedicated to the development and testing of a family of microcontrollers based on 8051 technology, like the 89C51 which we are going to use along this manual. You can download an evaluation which we are going to use along this manual. You can download an evaluation merely the same interface, this manual uses KEIL C51 $\mu$ Vision 3 with the C51 compiler v8.05a

\section{Ulti board:}

NI Ultiboard or formerly ULTIboard is an electronic Printed Circuit Board Layout program which is part of a suite of circuit design programs, along with NI Multisim. One of its major features is the Real Time Design Rule Check, a feature that was only offered on expensive work stations in the days when it was introduced. ULTIboard was originally created by a company named Ultimate Technology, which is now a subsidiary of National Instruments. Ultiboard includes a 3D PCB viewing mode, as well as integrated import and export features to the Schematic Capture and Simulation software in the suite, Multisim.
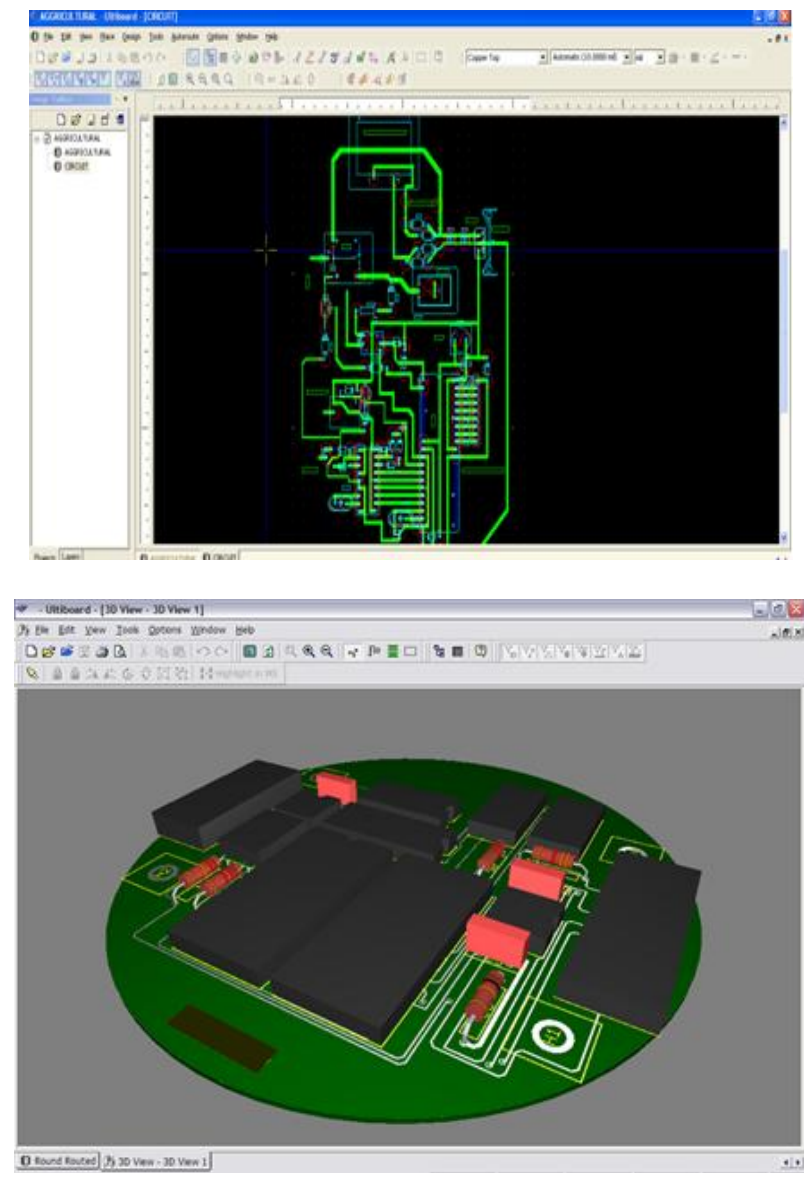

Fig6: ULTI board Screen shots 


\section{PCB MAKING:}

One of the most discouraging things about making a hardware project is building the printed circuit board-PCB.it is sometimes possible to use strip board or some other pre-fabricated board but more often than not the circuit complexity and performance requires a proper PCB to be made. The good news is that due to improvements in printing and processing technologies it is now relatively easy to make inexpensive high quality PCB's at home.

WARNING-Making PCB's requires the use of Ferric Chloride(FeCi3) which is corrosive so avoid skin and eye contact Remember safety-first so, use glasses, gloves and protective overalls .Ferric Chloride is also very good at distorting cloths weeks after you think you have washed it off. If you do get any on your skin then wash it off immediately with lots of water and soap.

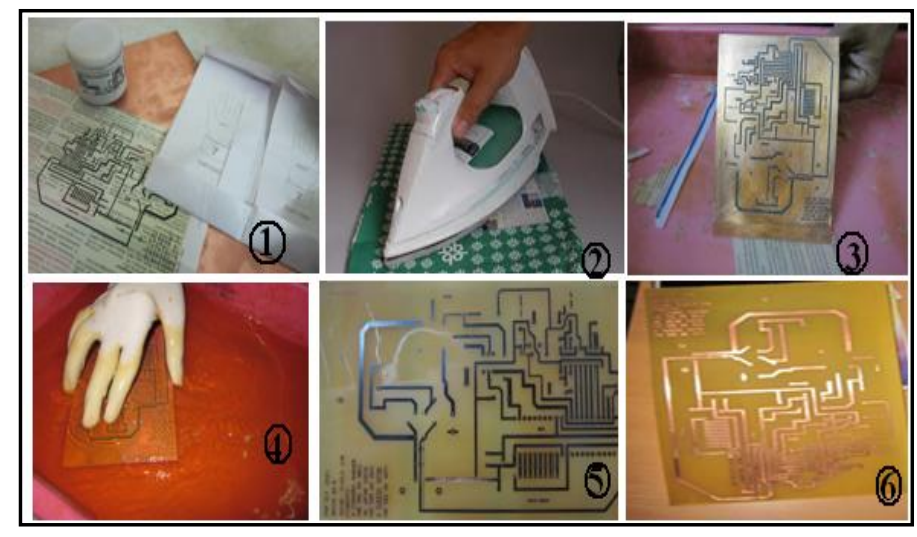

Fig7: PCB etching process

\section{ADVANTAGES}

* We can control the motor pump from any place of the world.

* Reduce the power consumption.

* Check the availability of power supply at pump set.

* Check the load on/off status by simply an IVR response.

* Set the particular period of time to on/off the motor pump.

* Password protection.

* Provides the information about rain at the field.

* Know the working hours of motor pump.

* Control the home appliances also.

\section{APPLICATIONS}

* Used in Agriculture Applications.

* Used in fields to control the motors.

* Used in home to control the Home Appliances.
6. RESULTS
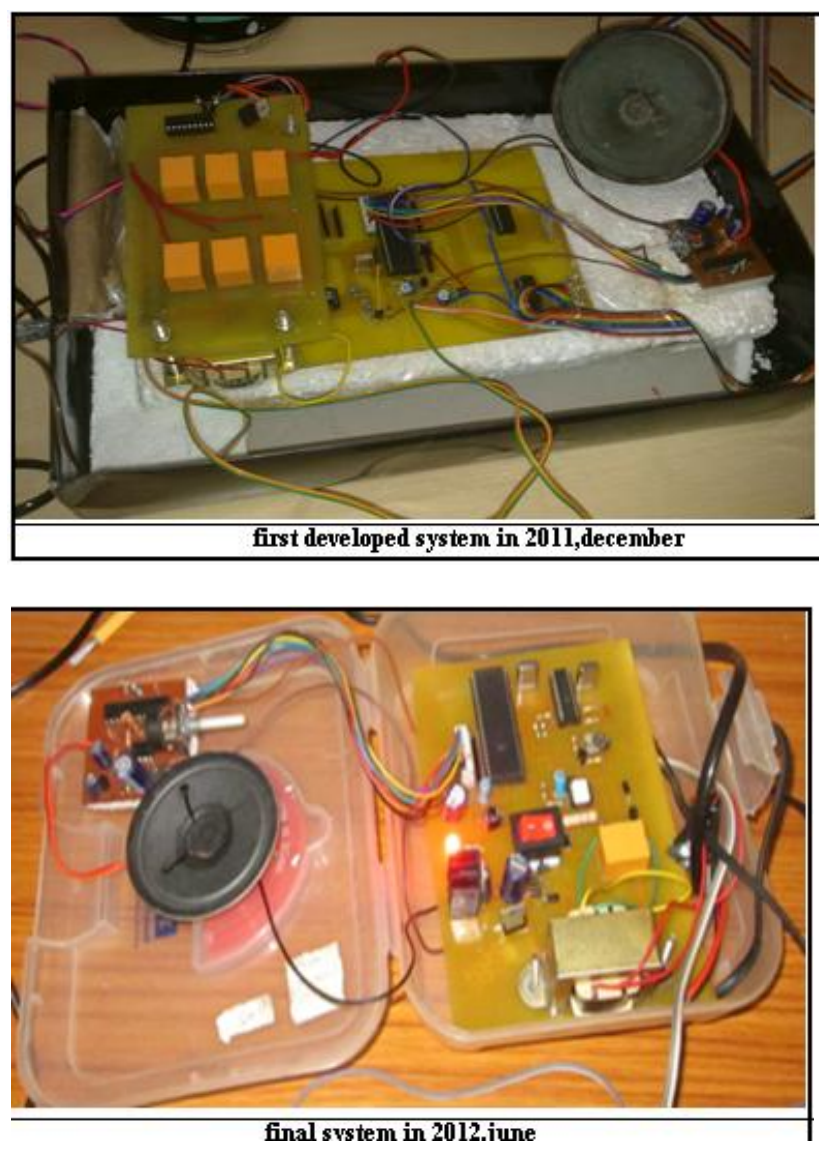

Fig8: Final system

\section{CONCLUSIONS}

As farmers can ensure timely water supply for their crops it will help them to make easy way and live a good life. The nnovation helps the formers get an equitable space in society.

\section{Benefits}

Save electricity

Low cost

Provide security

Easy way to operate.

\section{REFERENCES}

[1] The 8051 Microcontroller and Embedded Systems Using Assembly And $\mathrm{C}$ by Mazidi and Mazidi.

[2] Micro Controllers by Deshmukh, Tata McGraw Hill Edition.

[3] Embedded systems architecture, programming and design by rajkamal

[4] Microcontrollers (Theory \& Applications) by A.V. Deshmuk, WTMH, 2005. 
[5] D. J. Gardner-Bonneau, and H. Blanchard (ed), Human factors and voice interactive systems (Boston, USA: Kluwer, 2007)

[6] K. M. Lee \& J. Lai, Speech Versus Touch: A Comparative Study of the Use of Speech and DTMF Keypad for Navigation, International Journal of Human-Computer Interaction, 19 (3), 2005, 343-360.

[7] M.P. Plauche, U. Nallasamy, J. Pal, C. Wooters\&D.Ramachandran, Speech Recognition for Illiterate Access to Information and Technology, Proc. IEEE/ACM International Conferenceon Informationand Communication Technologies and Development (ICTD2006), USA, 2006.

[8] E. Brewer, M. Demmer, M. Ho, R.J. Honicky, J. Pal, M.Plauche, \& S. Surana. The Challenges of Technology Research for Developing Regions, IEEE Pervasive Computing, 5(2), 2006, 15-23.

[9] C. Wei \& B. Kolko, Studying mobile phone use in context: Cultural, political, and economic dimensions of mobilephoneuse, InternationalProfessional Communication Conference, Limerick, Ireland, July 10-13, 2005.

[10] C. Delogu, A.D. Carlo, P. Rotundi\& D. Sartori,Usability evaluation of IVR systems with DTMF and ASR,Proc. International Conference on Spoken Language Processing (ICSLP 98), Australia, 1998.

[11] T.S. Parikh \& E.D. Lazowska, Designing Architecture for Delivering Mobile Information Services to the Rural Developing World. Proc. 2006 International World Wide Web Conference (WWW 2006), 791 - 800, Scotland, 2006.

[12] Srinivasan J, the Role of Trustworthiness in Information Service Usage: The Case of Parry Information Kiosks, Tamil Nadu, India, Proc. IEEE/ACM International Conference on Information and Communication Technologies and Development (ICTD2007), India, 2007.

[13] N. Vijaykrishnan, M. Kandemir, M. Irwin, H. Kim, and W. Ye, "Energy-driven integrated hardware-software optimizations using Simple Power," in Proc. 27th Int. Symp. Computer Architecture, 2000,pp. 24-30.

[14] A. El Gamal and Z. A. Syed, "A stochastic model for interconnections in custom integrated circuits," IEEE Trans. Circuits Syst., vol. CAS-28,pp. 888-894, Sept. 1981.

[15] Workshop on Code Generation for Embedded Processors in Design Automation for Embedded Systems, vol. 4, Mar. 1999.

[16] http://en.wikipedia.org/wiki/Interactive_voice_response. 